\title{
Laparoscopic needle catheter jejunostomy by using a double semipurse string suture method in minimally invasive Ivor Lewis esophagectomy
}

\author{
Xuyang Peng ${ }^{1,2 \#}$, Xi Zhu ${ }^{3 \#}$, Zixiang Wu ${ }^{1}$, Qi Wang ${ }^{1}$, Shuai Fang ${ }^{1}$, Tianwei Zhan ${ }^{1}$, Ming Wu ${ }^{1}$ \\ ${ }^{1}$ Department of Thoracic Surgery, Second Affiliated Hospital, Zhejiang University School of Medicine, Hangzhou 310009, China; ${ }^{2}$ Department of \\ Cardiothoracic Surgery, Lishui People's Hospital, Lishui 323000, China; ${ }^{3}$ Department of Thyroid and Breast Surgery, Lishui Hospital of Zhejiang \\ University, Lishui Municipal Central Hospital, Lishui 323000, China \\ Contributions: (I) Conception and design: X Peng, M Wu; (II) Administrative support: X Peng; (III) Provision of study materials or patients: X Peng; \\ (IV) Collection and assembly of data: X Zhu, Z Wu; (V) Data analysis and interpretation: X Peng, M Wu, X Zhu, Z Wu; (VI) Manuscript writing: All \\ authors; (VII) Final approval of manuscript: All authors. \\ "These authors contributed equally to this work. \\ Correspondence to: Ming Wu, MD. Department of Thoracic Surgery, Zhejiang University School of Medicine, 88 Jiefang Road, Hangzhou 310009 , \\ China. Email: iwuming22@zju.edu.cn.
}

Background: To investigate the safety and effectiveness of a double semipurse string suture method for jejunum fixation in laparoscopic needle catheter jejunostomy in minimally invasive Ivor Lewis esophagectomy (MIILE).

Methods: Two hundred and six esophageal cancer patients continuously receiving MIILE from March 2014 to February 2018 were enrolled. In all patients, the double semipurse string suture method was applied for jejunum fixation in laparoscopic needle catheter jejunostomy. The methods and details of this technique are introduced herein. General information, clinical data, postoperative complications and follow-up results were retrospectively analyzed, and the complication causes and treatment methods are discussed.

Results: Laparoscopic needle catheter jejunostomy-using the double semipurse string suture method was successfully performed in 206 patients. The operative time of laparoscopic needle catheter jejunostomy was $10.56 \pm 2.04 \mathrm{~min}$. No conversion to laparotomy or postoperative death or serious infection associated with the jejunostomy tube occurred. The incidence of complications associated with the jejunostomy tube was $16.50 \%$ (34/206), and most of the complications were mild. Severe complications occurred in 2 cases $(0.97 \%)$, which were cured after reoperation, without serious consequence.

Conclusions: The double semipurse string suture method is safe, simple and feasible for the jejunum fixation in laparoscopic needle catheter jejunostomy in MIILE. It is worth popularization and clinical application.

Keywords: Double semipurse string suture; minimally invasive esophagectomy; Ivor Lewis; laparoscopic needle catheter jejunostomy

Submitted Aug 09, 2019. Accepted for publication Nov 12, 2019.

doi: $10.21037 /$ jtd.2020.01.53

View this article at: http://dx.doi.org/10.21037/jtd.2020.01.53 


\section{Introduction}

In upper gastrointestinal surgery, simultaneous jejunostomy and catheterization for postoperative enteral nutrition support have long been widely recognized and affirmed by medical community and physicians (1). In 2015, National Comprehensive Cancer Network (NCCN) guidelines also recommended jejunostomy for enteral nutrition support for patients with esophageal cancer (2). At present, an increasing number of physicians are choosing laparoscopic jejunostomy, especially laparoscopic needle catheter jejunostomy. In this technique, with the help of special puncture and catheterization devices, the jejunostomy operation is safer and more convenient, and the incidence of postoperative complications is lower (3). However, regardless of the method used, the complications associated with jejunostomy are still difficult to avoid. There is even a report that the secondary operation caused by jejunostomy has a high mortality rate (4). Therefore, many doctors worry about the management of catheter jejunostomy (5). The fixation of the jejunum on the abdominal wall is the key to the whole catheter jejunostomy (6). At present, there is a shortage of large sample size reports on continuously completed laparoscopic needle catheter jejunostomy. This study retrospectively analyzed the clinical data of 206 esophageal cancer patients who continuously received laparoscopic needle catheter jejunostomy by using the double semipurse string suture method in minimally invasive Ivor Lewis esophagectomy (MIILE).

\section{Methods}

\section{Subjects}

Two hundred and six esophageal cancer patients continuously receiving MIILE in our hospital from March 2014 to February 2018 were enrolled. In all patients, the double semipurse string suture method was used in laparoscopic needle catheter jejunostomy. All of the operations were performed by the same doctor. The jejunostomy duration was defined as lasting from the beginning of finding Treitz's ligament to the completion of stoma fixation. Each patient's basic information, clinical data and follow-up results were stored in a database and were managed and maintained by a special person. This study was approved by the ethics committee of Second Affiliated Hospital of Zhejiang University (2019-363). Written

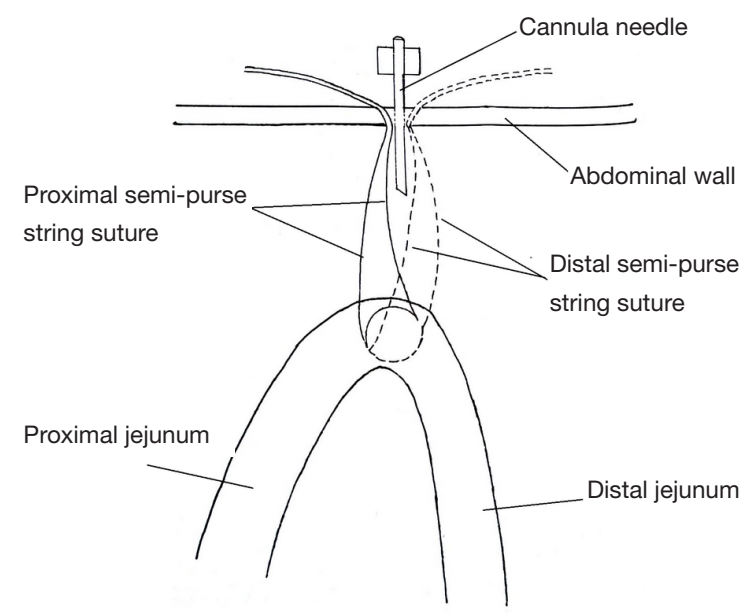

Figure 1 Layout of two semipurses at the jejunum.

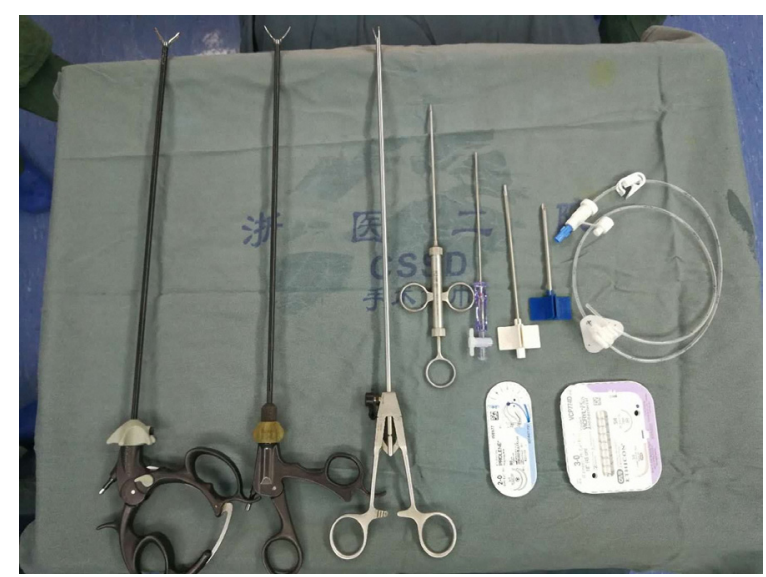

Figure 2 Freka ${ }^{\circledR}$-FKJ CH/FR9 jejunostomy devices and conventional laparoscopy apparatus.

informed consent was obtained from all participants.

\section{Surgical operation}

Laparoscopic needle catheter jejunostomy using the double semipurse string suture method was a part of laparoscopic operation in MIILE. The layout of two semipurses at the jejunum is shown in Figure 1. The surgical equipment included Freka ${ }^{\circledR}$-FKJ CH/FR9 jejunostomy devices (Fresenius Kabi AG, Bad Homburg, Germany) and conventional laparoscopy apparatus (Figure 2). Patients were in the supine position, followed by general intravenous 
anesthesia. A camera port with a diameter of $1.0 \mathrm{~cm}$ was made at a position $1 \mathrm{~cm}$ below the umbilicus. Two ports (diameter 10 and $5 \mathrm{~mm}$ ) were made at the right upper abdomen, and one port (diameter $5 \mathrm{~mm}$ ) was made at the left upper abdomen (Figure 3). Laparoscopic gastric mobilization and gastric conduit creation were performed, followed by laparoscopic needle catheter jejunostomy.

A position $20-30 \mathrm{~cm}$ from the Treitz ligament was used as a stoma site. Using the stoma site as the center, a seromuscular layer suture was performed at the proximal jejunum using 3-0 absorbable suture to form the first semipurse (Figure 4A). Then, a crochet hook was inserted to pull the suture out of the abdominal cavity (Figure $4 B$ )

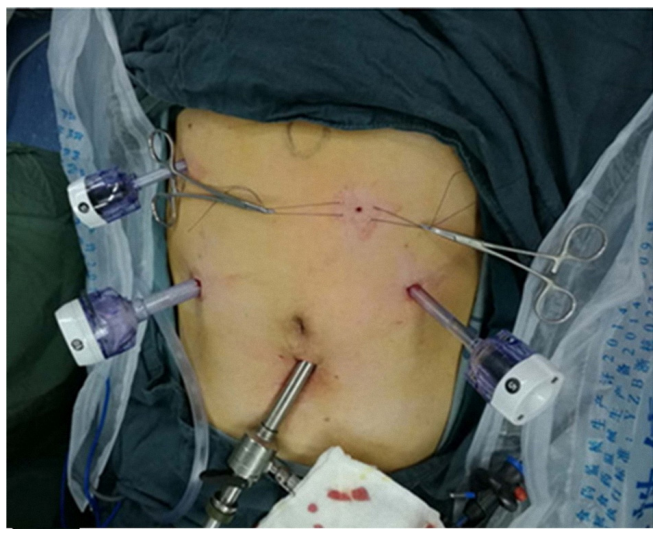

Figure 3 Distribution of surgical incisions. (performed by the assistant). The two ends of the suture were sutured to the subcutaneous position on one side of the puncture point using one empty needle (performed by the assistant). Using the same method, a second semipurse was made on the distal jejunum (Figure $4 C$ ), and the suture was pulled out of the abdominal cavity. The two ends of the suture were sutured to the subcutaneous position on the other side of the puncture point and sutured to the other side of the puncture point.

The tail of the semipurse string suture at the proximal jejunum was tightened to bring the proximal jejunum close to the abdominal wall. The cannula needle in the jejunostomy devices was inserted into the abdominal cavity along the original puncture point and was inserted into the jejunum cavity through the middle point of the two semipurses (Figure 4D). The position of the cannula needle in the jejunum cavity was confirmed using the needle core. The jejunostomy tube was inserted into the distal jejunum through the cannula needle. At the same time, normal saline was injected into the jejunum cavity in order to expand the jejunum cavity. The head end of the jejunostomy tube and its direction were tracked by the lens of the laparoscope. (Figure 4E).

After the jejunostomy tube was confirmed to be in the distal jejunum and the unobstructed, the cannula needle was cleavaged. The jejunostomy tube was pulled to the position corresponding with the $30 \mathrm{~cm}$ scale on the skin. The ends of the two semipurse string sutures outside the abdominal cavity were tightened to confirm that the
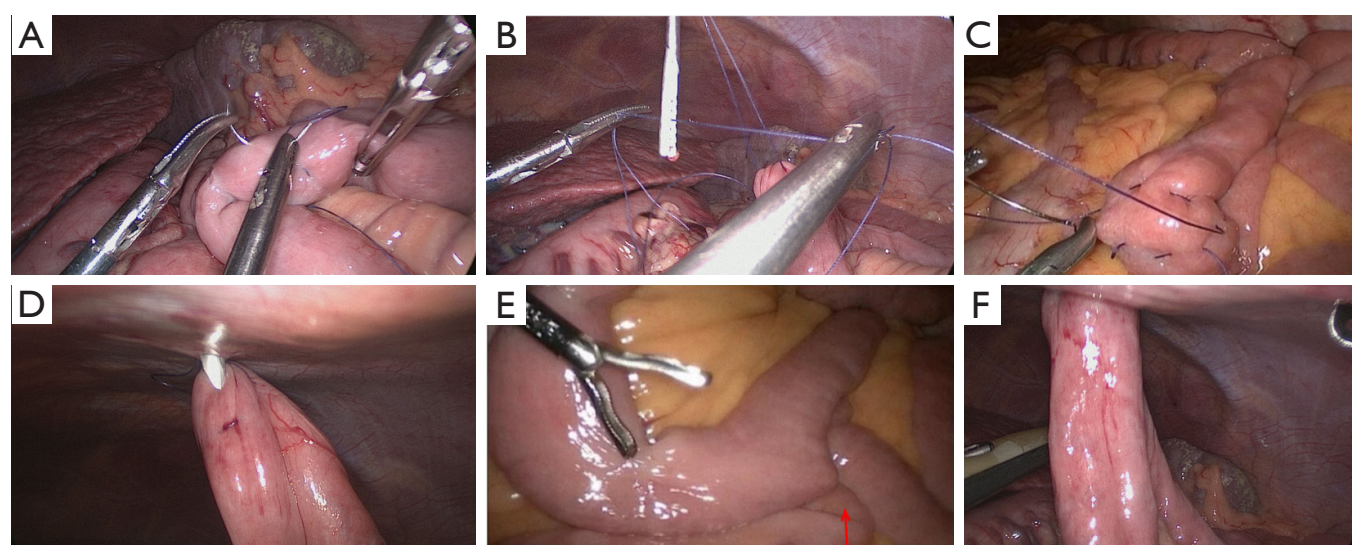

Figure 4 Intraoperative operation. (A) Making the first semipurse at the proximal jejunum; (B) pulling the suture out to abdominal cavity using crochet hook; (C) making the second semipurse at distal jejunum; (D) insertion of cannula needle into jejunum cavity through the middle point of double semipurse string sutures; (E) the jejunostomy tube had been inserted into the distal jejunum. The head end of jejunostomy tube was clearly visible in the jejunum cavity (the arrow indicated the head end of jejunostomy tube); (F) abdominal operation completion. 


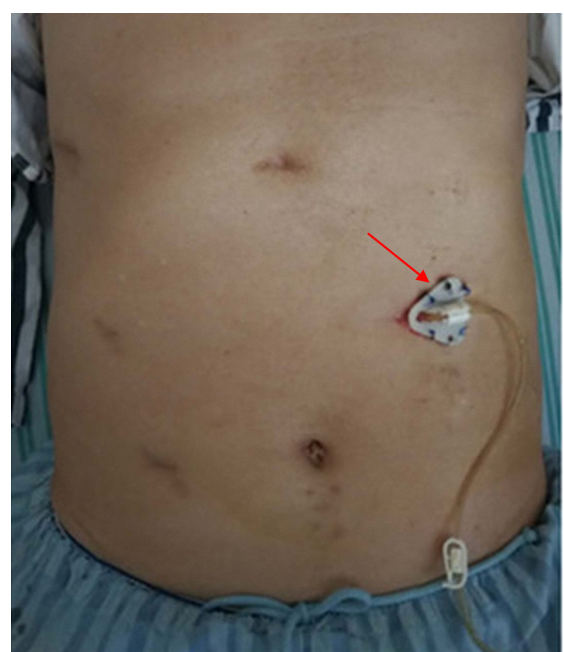

Figure 5 Condition of jejunostomy tube after 4 months from operation (the arrow indicated the fixation disc).

jejunum was close to the abdominal wall. The two sides of the intestinal climb were confirmed to be smooth and without volvulus. The tails of the semipurse string suture were knotted on the outside of the abdominal cavity. At this time, the laparoscopic surgery was completed (Figure 4F). The jejunostomy tube was placed in the special fixation disc. The fixation disc was sutured to the skin using a 2-0 proline suture (Figures 5, Video 1).

\section{Postoperative care and follow-up}

On the first day after the operation, $500 \mathrm{~mL}$ of $5 \%$ glucose solution or normal saline containing methylene blue was injected into the jejunostomy tube. The tightness between the jejunum stoma and abdominal wall was determined by observing the presence of methylene blue in the abdominal drainage. If there was no abnormality, the dose of enteral nutrition suspension [Nutricia Pharmaceutical (Wuxi) Co., Ltd., Wuxi, China] was gradually increased starting on the second day after operation. The target energy intake was $25-30 \mathrm{kcal} / \mathrm{kg}$ per day. The barium swallow method was performed on the 6th day after the operation to exclude the anastomotic leakage. If there was no abnormality, the oral diet intake was resumed on the 7 th day after operation. It was recommended that home enteral nutrition support be provided for at least 3 months after discharge (7). The first follow-up was performed within 2 weeks after discharge, followed by follow-up every 3 months throughout the first year. After that, follow-up
Table 1 Patient basic characteristics and postoperative clinical data

\begin{tabular}{|c|c|}
\hline Variable & $\mathrm{n}=206$ \\
\hline Age (years) & $64.61 \pm 8.12$ \\
\hline Sex (male:female) & 186:20 \\
\hline \multicolumn{2}{|l|}{ Tumor pathology, n (\%) } \\
\hline Squamous cell carcinoma & $184(89.32)$ \\
\hline Adenocarcinoma & $12(5.83)$ \\
\hline Others & $10(4.85)$ \\
\hline \multicolumn{2}{|l|}{ Tumor location, n (\%) } \\
\hline Middle esophagus & $86(41.75)$ \\
\hline Lower esophagus & $109(52.91)$ \\
\hline Gastro-esophageal junction & $11(5.34)$ \\
\hline Prior abdominal surgery, n (\%) & $27(13.11)$ \\
\hline Body mass index $\left(\mathrm{kg} / \mathrm{m}^{2}\right)$ & $21.80 \pm 2.70$ \\
\hline \multicolumn{2}{|l|}{ Tumor stage*, n (\%) } \\
\hline Stage $0 / 1$ & $42(20.39)$ \\
\hline Stage II & $75(36.41)$ \\
\hline Stage III & 89 (43.20) \\
\hline Operative time of catheter jejunostomy (min) & $10.56 \pm 2.04$ \\
\hline Postoperative hospitalization time (days) & $13.11 \pm 7.22$ \\
\hline Starting time of enteral nutrition support (days) & $1-3$ \\
\hline Retention time of jejunostomy tube (days) & $96.52 \pm 10.45$ \\
\hline
\end{tabular}

was conducted every six months.

\section{Results}

Laparoscopic needle catheter jejunostomy using the double semipurse string suture method was successfully performed in 206 patients with MIILE. By 4 months after the operation, the jejunostomy tube was fixed well. There was no obvious oozing or infection around the stoma in most cases (199 cases) (Figure 5). The patients' basic characteristics and postoperative clinical data are shown in Table 1. The operative duration of laparoscopic needle catheter jejunostomy was $10.56 \pm 2.04 \mathrm{~min}$. The starting time of enteral nutrition support after the operation was 1-3 days. Patients with anastomotic fistula or severe pulmonary infection were cured and discharged after conservative 
Table 2 Postoperative complications

\begin{tabular}{|c|c|}
\hline Complication & $\mathrm{N}(\%)$ \\
\hline \multicolumn{2}{|l|}{ Jejunostomy tube-associated complication } \\
\hline \multicolumn{2}{|l|}{ Intraoperative complication } \\
\hline Suture damage or fracture & $4(1.94)$ \\
\hline Conversion to laparotomy & 0 \\
\hline \multicolumn{2}{|l|}{ Postoperative complication } \\
\hline Gastrointestinal discomfort & $23(11.17)$ \\
\hline \multicolumn{2}{|l|}{ Mechanical complication } \\
\hline Dropped jejunal tube & $1(0.49)$ \\
\hline Cloggeded jejunal tube & $5(2.43)$ \\
\hline Bowel obstruction & $3(1.46)$ \\
\hline Volvulus & $1(0.49)$ \\
\hline Reoperation & $2(0.97)$ \\
\hline \multicolumn{2}{|l|}{ Infectious complication } \\
\hline Site oozing & $5(2.43)$ \\
\hline Site infection & $2(0.97)$ \\
\hline Jejunostomy tube-associated mortality & 0 \\
\hline Total incidence of complications & $34(16.50)$ \\
\hline \multicolumn{2}{|l|}{ Non-jejunostomy tube-associated complication } \\
\hline Anastomotic leak & $5(2.43)$ \\
\hline Pneumonia & $54(26.21)$ \\
\hline Atrial fibrillation & $45(21.84)$ \\
\hline Chylothorax & $5(2.43)$ \\
\hline In-hospital mortality & $1(0.49)$ \\
\hline
\end{tabular}

treatment with the help of prolonged enteral nutrition support. The average length of postoperative hospitalization time of all patients was $13.11 \pm 7.22$ days. The average retention time for jejunostomy tubes was $96.52 \pm 10.45$ days.

The incidence of complications associated with jejunostomy tubes was $16.50 \%(34 / 206)$, and most complications were mild (Table 2). No bleeding, perforation or conversion to laparotomy occurred during the operations. No postoperative complications such as dislodged jejunostomy tube, jejunal-cutaneous fistula, bowel necrosis, herniation, jejunostomy tube breakage or severe infection occurred. One patient died of sudden massive acute myocardial infarction. There were no postoperative deaths associated with jejunostomy tubes.
Severe complications occurred in 2 cases $(0.97 \%)$, which were cured after reoperation, without serious consequence. One case developed a clogged jejunostomy tube on the 8th day after operation, and treatment by saline irrigation was ineffective. The jejunostomy tube was replaced in reoperation. Another case had an acute intestinal obstruction caused by volvulus on the 10th day after operation. A volvulus restoration combined with an intestinal adhesion release was promptly performed, and no intestinal necrosis occurred.

There were a total of 5 cases $(2.43 \%)$ of clogged jejunostomy tube after operation. Except for 1 case which needed reoperation to replace the jejunostomy tube, the other 4 cases underwent recanalization after saline irrigation. Five cases $(2.43 \%)$ had repeated oozing around the stoma after operation. Two cases $(0.97 \%)$ had purulent secretions in superficial skin around the stoma, but were completely recovered after repeated wound dressing. There was 1 case $(0.49 \%)$ of dropped jejunostomy tube in which the jejunostomy tube was accidentally removed at home 6 weeks after the operation, but no serious consequence occurred. After the operation, 23 cases $(11.17 \%)$ had gastrointestinal discomfort. The main symptoms were diarrhea and abdominal distention. After adjusting the infusion amount, speed, and temperature of the enteral nutrition solution, and using related drugs when necessary, all patients experienced relief, and could tolerate the enteral nutrition support treatment.

In this study, the learning curve of the double semipurse string suture method was investigated (Figure 6). It was found that the operative time of catheter jejunostomy was basically stable after approximately 20 operations were completed. In addition, 2 patients with severe complications were within the first 20 cases. Therefore, the accumulation of experience from approximately 20 cases was needed to skillfully and safely master this technique.

\section{Discussion}

In 1990, O'Regan and Scarrow first reported laparoscopic jejunostomy (7). Since then, with the rapid development of minimally invasive surgery and the progress of surgical instruments, the methods of laparoscopic jejunostomy have emerged in an endless stream. To improve the safety and maneuverability of the surgery, reducing the complications associated with jejunostomy is required. In recent years, many improved methods of laparoscopic jejunostomy have been reported $(8,9)$. It is well known that in most cases of 


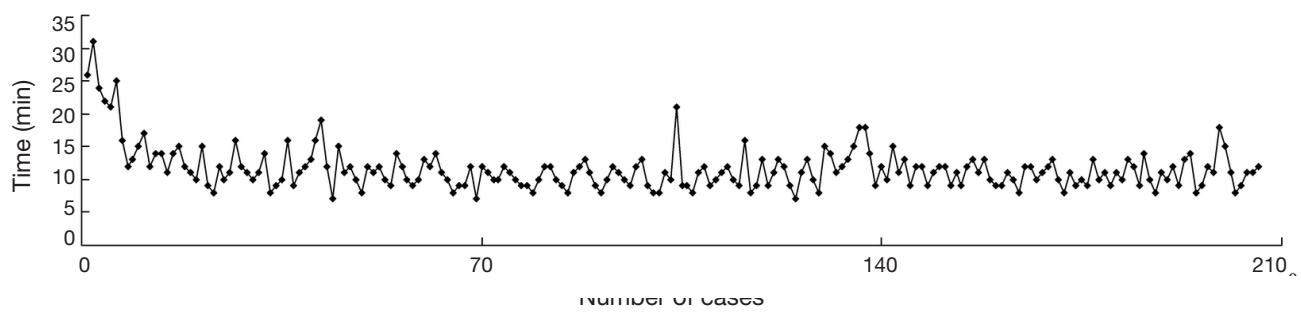

Figure 6 Learning curve of double semipurse string suture method.

laparoscopic jejunostomy, the jejunostomy tube needs to be placed in the jejunum and fixed on the abdominal wall. It is found that the fixation of jejunum on abdominal wall can cause serous fibrinous and suppurative fibrinous exudation, leading to the risk of postoperative intestinal adhesion and intestinal obstruction. If the jejunum and abdominal wall are not fixed properly, postoperative intestinal fistula, peritonitis, abdominal wall infection and other serious complications will occur and require reoperation (6). Therefore, it is believed that the fixation of the jejunum on abdominal wall is the key to the whole laparoscopic jejunostomy.

Commonly used jejunum fixation methods in jejunostomy include the intraperitoneal suture, transabdominal suture, and T-fastener. In the initial stage of the laparoscopic jejunostomy, we used the intracorporeal suture as the fixation method. This method requires many sutures and knotting operations under the endoscope. The operation takes a long time, and the operation field is not well exposed in the suture process. The requirement of the surgeon's endoscopic technique is high, and the learning curve is long. In recent years, it has been reported that the efficiency of endoscopic suture can be improved with the aid of some special instruments such as V-Loc (10) and Endostitch (11). However, these special suture instruments have not been adopted by us due to the high price. Considering the complexity of radical surgery for esophageal cancer and the long duration of the operation itself, we do not want to spend too much time in jejunostomy. Since then, the endoscopic transabdominal suture is used for fixation of the jejunum and the abdominal wall. Compared with the intraperitoneal suture, the greatest advantages of this method are the simplified operation process and shortened operation time. To make up for the deficiency of intraperitoneal suture, the arrangement method of fixation suture on the jejunum surface is amended, and the double semipurse string suture method is applied.
The advantages of the double semipurse string suture method are very obvious. First, the function of the double semipurse string suture is close to the purse string suture. After suture knotting and fixation, the tightness between jejunum and abdominal wall is good, and no extra sutures are needed. Second, as only a semipurse string suture is needed for each needle, the change of the suture needle insertion angle is small, and the operation is easy. The whole operation is completed under the direct view of the endoscope, and the operation field is well exposed. Third, the number of fixation sutures is reduced from 4 originally to 2, and no knotting under endoscope is needed, which greatly reduces operation time, and makes the operation easier for the surgeon to learn to master. The jejunostomy operation time is only a very small part of the whole esophagus cancer operation. Fourth, there is no serious infectious complication or dislodged jejunostomy tube.

This technique also has some limitations. During the operation process, both the crochet hook and cannula needle work in the same needle path. When the second semipurse string suture is pulled out of the abdominal wall, the first semipurse string suture that already exists in the abdominal wall may be damaged. Therefore, some scholars advocate the intraperitoneal suture and fixation, which is believed to reduce the risk of early catheter intraperitoneal displacement in the early postoperative period (12). In our experience, when encountering greater resistance in the puncture process, the crochet hook may hook the suture. Therefore, the needling angle should be promptly adjusted. To reduce the probability of the puncturing process damaging the suture, a different peritoneum puncture point can be selected when the crochet hook enters the abdominal cavity the second time. If there suspected suture damage, it is recommended that the suture be replaced for the secondary suture. We think that inspection after catheterization completion can avoid the occurrence of the above problems. In addition, the condition of the sutures 
should be checked again before closing the abdomen.

Another important task during the operation is to ensure the correct position of the jejunostomy tube. A previous study (13) has shown that nearly half of reoperation after jejunostomy can be avoided by properly fixing the jejunostomy tube and ensuring its normal location. We recommend the injection of normal saline into the jejunostomy tube to expand the jejunum cavity. Based on this, the activity of the jejunostomy tube in the jejunum cavity is clear, and it is easy to put the jejunostomy tube into the distal jejunum. We have tried expanding the intestinal tract by injecting air, but the air cannot dissipate in a short time after injection. The patient is prone to abdominal distention after operation, which affects the recovery of postoperative intestinal function. Therefore, it is rarely used now.

The most common complication after jejunostomy is gastrointestinal symptoms. This is mainly due to the shortterm intolerance of the intestinal tract to the hypertonic enteral nutrition solution, and it is not related to the jejunostomy operation itself. The most common symptoms are diarrhea and abdominal distention. According to the literature $(14,15)$, the incidence of these symptoms is $5-35 \%$. In this study, there were 23 cases ( $11.17 \%)$ with postoperative gastrointestinal discomfort, which is basically consistent with the above reports. Although the mechanical complications are relatively rare, they are often more severe and may even require reoperation. The clogged jejunostomy tube is the most common mechanical complication. In this study, we found that the crystallization of the drug in the jejunostomy tube was the main cause of clogged jejunostomy tube. There was 1 patient with clogged jejunostomy tube that could not be solved by recanalization, so a reoperation was performed. We think that mastering the standardized administration method (e.g., flushing jejunostomy tube immediately after drug administration; no injection of insoluble drugs; strengthening the jejunostomy tube related follow-up) through the jejunostomy tube can reduce the risk of clogged jejunostomy tube. Therefore, the training of nursing staffs needs to be strengthened.

In this study, there was 1 case of postoperative acute intestinal obstruction caused by volvulus, which required reoperation (relaparotomy). Although some scholars believe that postoperative intestinal volvulus is related to a digestive tract abnormality (16), it is undeniable that the intestinal volvulus has a certain relationship with the operation itself. As found in the reoperation in this case, there was an obvious angulation of the intestinal climbs on both sides of stoma after adhesion. We think that this has a certain relationship with the occurrence of volvulus. It is suggested that the intestinal ligation should be added to prevent the postoperative intestinal volvulus. The usual practice is to suture the jejunum wall with the distance $3-4 \mathrm{~cm}$ from stoma on the abdominal wall, to eliminate the angulation of the intestinal climbs. However, this is not a conventional surgical procedure and is mainly dependent on the surgeon's judgment and experience. In this study, there were 2 cases of incomplete intestinal obstruction after operation which were relieved after conservative treatment.

Wound infection is also one of the common complications of jejunostomy. In this study, it was found that most of the patients with oozing and infection after operation have long retention time of the jejunostomy tube, and they were undergoing postoperative adjuvant chemotherapy. Long jejunostomy tube retention time may increase the risk of infection, but the long duration of home enteral nutrition support can help patients to persist in postoperative radiotherapy and chemotherapy. Therefore, we propose the simultaneous jejunostomy and home enteral nutrition support. We recommend that home enteral nutrition support is provided for at least 3 months after discharge (17). In this study, the oozing and infection were able to be completely alleviated through simple wound dressing. Therefore, these patients generally benefited from the long duration of jejunostomy tube retention.

In this study, there were five patients with postoperative chyle leaks. It is believed that the early enteral nutrition may increase the incidence of chyle leaks after esophageal cancer surgery. However, in this study, the incidence of chyle leaks after operation is even slightly lower than that in reported studies $(18,19)$. Due to the routine prophylactic ligation of thoracic duct, all patients with chyle leaks can achieve successful outcome through conservative treatment. Therefore, we still advocate the early enteral nutrition after esophageal cancer surgery.

It is reported that the incidence of reoperation after jejunostomy is $1.0-1.8 \%(13,20,21)$. In this study, the incidence of reoperation was within $1 \%$. Most of the complications associated with jejunostomy tubes were mild, without serious consequences. This is a surprising result, which is enough to indicate the safety of this technique. However, this study is a retrospective analysis, lacking information from a control group. Compared with a prospective, controlled study, there is a certain information bias in this study. In addition, all subjects enrolled in this study are patients with esophageal cancer which can be surgically excised. Patients who had lost 
opportunity for surgery and had undergone palliative chemotherapy after simple jejunostomy were not included in this study. In these excluded patients the disease is more complex, and nutritional status is worse, so the possibility of complications associated with jejunostomy tubes may increase. Nevertheless, due to the large sample size, this study still has certain clinical reference value.

\section{Conclusions}

In laparoscopic needle catheter jejunostomy in MIILE, the double semipurse string suture method is a safe, simple and feasible technique for jejunum fixation. The incidence of postoperative complications associated with jejunostomy tubes and reoperation is low. This method is worth popularizing and clinical application.

\section{Acknowledgments}

Funding: None.

\section{Footnote}

Conflicts of Interest: The authors have no conflicts of interest to declare.

Ethical Statement: The authors are accountable for all aspects of the work in ensuring that questions related to the accuracy or integrity of any part of the work are appropriately investigated and resolved. This study was approved by the ethics committee of Second Affiliated Hospital of Zhejiang University (2019-363). Written informed consent was obtained from all participants.

Open Access Statement: This is an Open Access article distributed in accordance with the Creative Commons Attribution-NonCommercial-NoDerivs 4.0 International License (CC BY-NC-ND 4.0), which permits the noncommercial replication and distribution of the article with the strict proviso that no changes or edits are made and the original work is properly cited (including links to both the formal publication through the relevant DOI and the license). See: https://creativecommons.org/licenses/by-nc-nd/4.0/.

\section{References}

1. De Gottardi A, Krähenbühl L, Farhadi J, et al. Clinical experience of feeding through a needle catheter jejunostomy after major abdominal operations. Eur J Surg 1999;165:1055-60.

2. Ajani JA, D'Amico TA, Almhanna K, et al. Esophageal and esophagogastric junction cancers, version 1.2015. J Natl Compr Canc Netw 2015;13:194-227.

3. Sica GS, Sujendran V, Wheeler Soin B, et al. Needle catheter jejunostomy at esophagectomy for cancer. J Surg Oncol 2005;91:276-9.

4. Han-Geurts IJM, Verhoef C, Tilanus HW. Relaparotomy following complications of feeding jejunostomy in esophageal surgery. Dig Surg 2004;21:192-6.

5. Date RS, Clements WDB, Gilliland R. Feeding jejunostomy: is there enough evidence to justify its routine use? Dig Surg 2004;21:142-5.

6. Gong L, Yan B, Chen Y, et al. Alternative method for jejunostomy in Ivor-Lewis esophagectomy. Thorac Cancer 2015;6:296-302.

7. O'Regan PJ, Scarrow GD. Laparoscopic jejunostomy. Endoscopy 1990;22:39-40.

8. Ye P, Zeng L, Sun F, et al. A new modified technique of laparoscopic needle catheter jejunostomy: a 2-year followup study. Ther Clin Risk Manag 2016;12:103-8.

9. Siow SL, Mahendran HA, Wong CM, et al. Laparoscopic T-tube feeding jejunostomy as an adjunct to staging laparoscopy for upper gastrointestinal malignancies: the technique and review of outcomes. BMC Surg 2017;17:25.

10. Yang SM, Hsiao WL, Lin JH, et al. Laparoscopic percutaneous jejunostomy with intracorporeal V-Loc jejunopexy in esophageal cancer. Surg Endosc 2017;31:2678-86.

11. Pennathur A, Awais O, Luketi ch JD. Technique of minimally invasive Ivor Lewis esophagectomy. Ann Thorac Surg 2010;89:S2159-S2162.

12. Young MT, Troung H, Gebhart A, et al. Outcomes of laparoscopic feeding jejunostomy tube placement in 299 patients. Surg Endosc 2016;30:126-31.

13. Myers JG, Page CP, Stewart RM. Complications of needle catheter jejunostomy in 2,022 consecutive applications. Am J Surg 1995;170:547-50; discussion 550-1.

14. Kight CE. Nutrition consideration in esophagectomy patients. Nutr Clin Pract 2008;23:521e8.

15. Han-Geurts IJ, Hop WC, Verhoef C, et al. Randomized clinical trial comparing feeding jejunostomy with nasoduodenal tube placement in patients undergoing oesophagectomy. Br J Surg 2007;94:31-5.

16. Belsha D, Thomson M, Dass DR, et al. Assessment of the safety and efficacy of percutaneous laparoscopic endoscopic jejunostomy (PLEJ). J Pediatr Surg 2016;51:513-8. 
17. Wu Z, Wu M, Wang Q, et al. Home enteral nutrition after minimally invasive esophagectomy can improve quality of life and reduce the risk of malnutrition. Asia Pac J Clin Nutr 2018;27:129-36.

18. Shah RD, Luketich JD, Schuchert MJ, et al.

Postesophagectomy chylothorax: incidence, risk factors, and outcomes. Ann Thorac Surg 2012;93:897-903; discussion 903-4.

19. Brinkmann S, Schroeder W, Junggeburth K, et al. Incidence and management of chylothorax after Ivor Lewis esophagectomy for cancer of the esophagus. J Thorac Cardiovasc Surg 2016;151:1398-404.

20. Ryan AM, Rowley SP, Healy LA, et al. Postoesophagectomy early enteral nutrition via a needle catheter jejunostomy: 8-year experience at a specialist unit. Clin Nutr 2006;25:386-93.

21. Han-Geurts IJ, Lim A, Stijnen T, et al. Laparoscopic feeding jejunostomy: a systematic review. Surg Endosc 2005;19:951-7.

Cite this article as: Peng X, Zhu X, Wu Z, Wang Q, Fang S, Zhan T, Wu M. Laparoscopic needle catheter jejunostomy by using a double semipurse string suture method in minimally invasive Ivor Lewis esophagectomy. J Thorac Dis 2020;12(3):240248. doi: $10.21037 /$ jtd.2020.01.53 